\title{
TWO-DIMENSIONAL MAGNETOEXCITONS IN THE FRACTIONAL QUANTUM HALL REGIME
}

\begin{abstract}
(Box 530, SE-751 21, Uppsala, Sweden)
${ }^{4}$ Moldova State University

PACS 71.35.Lk, 67.85.Jk (C) 2011

rons and hole layers are spatially separated, and the Coulon electron-hole interaction diminishes, a two-dimensional electron gas (2DEG) and a two-dimensional hole gas (2DHG) are formed. Their properties under conditions of the fractional quantum Hall effect can influence the properties of $2 \mathrm{D}$ magnetoexcitons. These properties are discussed in the present review.
\end{abstract}

S.A. MOSKALENKO,${ }^{1}$ M.A. LIBERMAN, ${ }^{2}$ B.V. NOVIKOV, ${ }^{3}$ E.S. KISELIOVA, ${ }^{4}$ E.V. DUMANOV ${ }^{1}$ F. CERBU $^{1}$

${ }^{1}$ Institute of Applied Physics, Academy of Sciences of Moldova

(5, Academic Str., Chisinau MD2028, Republic of Moldova)

${ }^{2}$ Departmant of Physics, Uppsala University

${ }^{3}$ Department of Solid State Physics, Institute of Physics, St.-Petersburg State University (1, Ulyanovskaya Str., Petrodvorets, St.-Petersburg 198504, Russia)

(60, A. Mateevici Str., Chisinau 2012, Republic of Moldova) outstanding scientific achievements in exciton physics. sary of his birthday.
Soviet Union, the highest distinction in USSR, for their

The present review was written in the honor of Academician M.S. Brodin celebrating the eightieth anniver-

\section{Introduction}

The theory of two-dimensional (2D) magnetoexcitons was developed in a number of papers including the investigations of their Bose-Einstein condensation, optical properties, and collective elementary excitations [1-8]. The actual developments in exciton physics are based on the previous achievements in this field, including the contribution of the Institute of Physics (IOP) of the National Academy of Science of Ukraine (NASU) situated in Kiev, where, due to the initiative of Academicians A.S. Davydov and A.F. Prikhot'ko, the investigations in the field of exciton physics were organized on a large scale many years ago. It is sufficient to mention the group of that time collaborators and of former collaborators at the IOP NASU in the composition of A.S. Davydov, A.F. Prikhot'ko, M.S. Brodin, V.L. Broude, A.F. Lubchenko and E.I. Rashba together with their colleagues E.F. Gross, A.A. Kaplyanskii, and B.P. Zakharchenya from A.F. Ioffe Physico-Tehnical Institute of the Russian Academy of Sciences. In 1966, all of them were awarded by the Lenin prize of the former

\section{The Ginzburg-Landau Theory of the Fractional Quantum Hall Effect}

Describing these questions, we will follow the collective monograph [9] devoted to the fractional quantum Hall effect (FQHE) and the candidatus scieritiarum thesis written by Enger [10] in a clear and accessible way, as well as many published papers cited below. The Landau theory for the second-order phase transition is based on the introduction of an order parameter [11] $\phi(r t)$, by assuming that the free energy is a regular function of $\phi$ at least near the critical point. In the case of superconductors and superfluids, the role of order parameter is played by the condensate wave functions. The theory was elaborated by Ginzburg and Landau [12] for superconductors and by Ginzburg and Pitaevskii [13] for liquid helium. The microscopic foundations in the latter case were proposed by Pitaevskii [14] and Gross [15] and were explained in the monograph by Nozieres and Pines [16]. The microscopic theory of superfluidity was firstly proposed by Bogoliubov in the model of weakly interacting Bose gas [17]. The density of the Helmholz free energy $f(r)$ expanded in the small order parameter $\phi$ has the form

$f(r)=f_{0}+\alpha|\phi|^{2}+\frac{\beta}{2}|\phi|^{4}+\frac{\hbar^{2}}{2 m}|\nabla \phi|^{2}$ 
In the case of superconductors, it is necessary to include the effect of an applied electromagnetic field. This can be made, by substituting the canonical momentum $\hat{p}=$ $-i \hbar \nabla$ by the kinetic momentum

$\mathbf{p}-\frac{q}{c} \mathbf{A}(r)$,

where $\mathbf{A}$ is the vector potential, and $q$ is the charge of a Cooper pair, $q=-2 e$.

The density of the Gibbs free energy including also the density of the magnetic field energy looks as

$g(r)=f_{0}+\alpha|\phi|^{2}+\frac{\beta}{2}|\phi|^{4}+$

$+\frac{1}{2 m}\left|\left(-i \hbar \nabla+\frac{2 e}{c} \mathbf{A}\right) \phi\right|^{2}+\frac{B^{2}}{2 \mu_{0}}$,

where $\mathbf{B}=\operatorname{rot} \mathbf{A}$. Minimizing the total Gibbs energy $G=\int g(r) d r$ with respect to $\phi$ and $\mathbf{A}$ gives

$\frac{1}{2 m}\left(-i \hbar \nabla+\frac{2 e}{c} \mathbf{A}\right)^{2} \phi+\alpha \phi+\beta|\phi|^{2} \phi=0$,

$\frac{1}{\mu_{0}} \nabla \times \mathbf{B}=\frac{i e \hbar}{m}\left(\phi^{*} \nabla \phi-\phi \nabla \phi^{*}\right)-\frac{4 e^{2}}{m c^{2}}|\phi|^{2} \mathbf{A}$.

They are the Ginzburg-Landau (GL) equations, where $\mu_{0}$ is the magnetic permeability.

The Ginzburg-Pitaevskii-Gross equation for the Bose-Einstein condensate wave function $\phi(r, t)$ is

$i \hbar \frac{\partial \phi(r, t)}{\partial t}=-\frac{\hbar^{2}}{2 m} \Delta \phi(r, t)+\lambda|\phi(r, t)|^{2} \phi(r, t)$.

Separating the space and time parts $\phi(r, t)=e^{-i \mu t} \phi(r)$, and choosing the chemical potential $\mu=\lambda \rho_{0}$, one can transform (5) into the equation

$-\frac{\hbar^{2}}{2 m} \Delta \phi(r)+\lambda\left(|\phi(r)|^{2}-\rho_{0}\right) \phi(r)=0$,

which is known as Gross-Pitaevskii equation or nonlinear Schrödinger equation. As was mentioned in $[9$, 18 , the GL theory is needed also for the FQHE to better understand this phenomenon.

The FQHE is a remarkable example of the quantum effects observable on a macroscopic level similarly as superconductivity and superfluidity. All these phenomena have a ground state with a non-zero density of particles. In all three cases, there are quasiparticle excitations in the form of vortices. But there are some aspects of the
FQHE, which are not present in the GL theories of superconductors and superfluids. First, there is a gap in the spectrum of collective elementary excitations, which leads to the incompressibility of the FQHE systems. The second important difference is related to the properties of vortices in the FQHE case. They play the role of single-particle excitations and have a finite creation energy, as opposite to the vortices in the superfluid He-II with an extensive creation energy of a vortex proportional to $\ln (R / a)$, where $R$ is the radius of the system and $a$ is the vortex core.

In addition, the FQHE vortices have fractional charges [18]. In a number of papers, some versions of the GL theory for the FQHE were proposed starting with the Lagrangian of the system containing the supplementary term known as Chern-Simons term. It describes the gauge vector potential generated by the vortices; they are induced, in turn, by flux quanta created by the external magnetic field $B$. Instead of the Gibbs free energy, the action of the system is studied.

Girvin [18] and Girvin and MacDonald [19] proposed, for the first time, a phenomenological version of the GL theory, by writing the action $S$ in the form

$S=\int d^{2} r\left\{\left|\left(-i \hbar \nabla+\frac{e}{c} A_{1}(r) \psi(r)\right)\right|^{2}+\right.$

$+i\left(\psi^{*}(r) \psi(r)-n_{0}\right) \phi(r)-$

$\left.-\frac{i \theta}{8 \pi^{2}}\left(\phi \nabla \times \mathbf{A}_{1}+\mathbf{A}_{1} \times \nabla \phi\right)\right\}$

where

$\mathbf{A}_{1}=\mathbf{A}+\mathbf{a}$

is an effective summary vector potential composed from the physical external vector potential $\mathbf{A}$ generating the magnetic field $\mathbf{B}=\operatorname{rot} \mathbf{A}$ and from a gauge vector potential a created by the vortices. The effective field $A_{1}$ represents the frustration arising in the system, when the density of particles $\rho(r)=|\psi(r)|^{2}$ deviates from the quantized Laughlin's density $n_{0}$ [20], which determines the fractional filling factor $\nu=1 / m$ with $m$ integer. The density $n_{0}$ is named the flux density being determined by the magnetic field $\mathbf{B}$ through the magnetic length $l$ in the form $n_{0}=1 / m 2 \pi l^{2}$, where $l^{2}=\hbar c / e B$. The equation of motion for the vector $\mathbf{A}_{1}$ in a static case looks as

$\theta \nabla \times \mathbf{A}_{1}=\left(\psi^{*} \psi-n_{0}\right), \quad \theta=2 \pi / m$. 
The proposed phenomenological GL theory allows one to understand that the creation energy of a single vortex is finite, and that the vortex has a fractional charge. The difference between the FQHE and ordinary superfluidity was explained by the strong phase fluctuations induced by the frustration.

Zhang, Hansen, and Kivelson [20] derived their fieldtheory model starting from the microscopic Hamiltonian. They constructed the GL theory similar to that by Girvin, but with one difference concerning the ChernSimons term, which contains only the gauge field $a(r)$ [20]. As in the previous papers [19], it was confirmed that the disturbances of the localized density moving the system away from the good filling fractions lead to the creation of single-particle excitations. These quasiparticle and quasihole excitations have the form of vortices with static nonuniform finite-energy solutions. Side by side with the single-particle excitations, the collective elementary excitations were discussed in [20]. To this end, the Lagrangian was expanded up to terms quadratic in $\delta \phi$ and $\delta a$ about the constant solutions corresponding to vacuum expectation values. The fluctuating values $\delta \phi$ and $\delta a$ were represented in the form of plane waves with vector $\mathbf{q}$. The dispersion relation was found in the form [20]

$\omega^{2}(q)=(e \kappa B)^{2}+\frac{1}{4} \kappa q^{2}\left(\kappa q^{2}+8 \lambda n_{0}\right)$.

It has a gap at the point $q=0$ proportional to the external magnetic field $B$. For negative $\lambda$, but for a sufficiently small parameter $|\lambda| / \kappa$, the dispersion curve has a roton-type behavior with the same shape, as was derived by Girvin, MacDonald, and Platzman [21]. The GL theory developed in [20] describes the incompressibility, fractional charge, and fractional statistics of quasiparticles. But, being a coarse-grained version of the FQHE, it makes errors on the magnetic length scale. It treats the gauge field in the mean-field approximation and reproduces correctly the long-wavelength effects of the quantum Hall systems excluding such details as the description of a vortex core. The idea that the long-wavelength effects of the physical magnetic field are cancelled by the gauge field was also suggested by Laughlin [22] and in $[23,24]$.

\section{Point Vortices in the Conditions of FQHE}

Because the vortices play an important role in the understanding of the FQHE, some information about them is needed. The explanations presented below beginning with classical hydrodynamics and going on to quantum vortices have been gotten from Enger [10] and Myklebus [25] theses. An ideal fluid without viscosity is described in classical hydrodynamics by the continuity equation

$\frac{\partial \rho}{\partial t}+\nabla(\rho \mathbf{v})=0$

and Euler's equation

$\frac{\partial \mathbf{v}}{\partial t}+(\mathbf{v} \cdot \nabla) \mathbf{v}=-\frac{\nabla p}{\rho}$

where $\rho, p$, and $\mathbf{v}$ are the density, pressure, and velocity field, respectively, at each point of the liquid. The vorticity is defined in $3 \mathrm{D}$ hydrodynamics as $\boldsymbol{\omega}=\boldsymbol{\nabla} \times \mathbf{v}$. If the liquid is not only ideal but also isentropic, with constant entropy along it, the vorticity $\boldsymbol{\omega}$ obeys a supplementary continuity equation. If $\boldsymbol{\omega}=0$ at all points of the fluid, it is called irrotational with a potential flow. In this case, one may introduce the velocity potential $\phi$

$\mathbf{v}=\boldsymbol{\nabla} \phi, \quad \boldsymbol{\omega}=\boldsymbol{\nabla} \times \mathbf{v}=0$.

In physical fluids, the vorticity is localized in small areas. Outside of the vortices, the majority of a fluid is irrotational. In a $3 \mathrm{D}$ liquid, the vortex is a tube with strength $\kappa$ defined as

$\kappa=\int \boldsymbol{\omega} d \boldsymbol{\sigma}=\oint \mathbf{v} d \mathbf{l}$

The Helmholtz theorem establishes that the same particles take part in the vortex formation in all the time and that the strength is the same in all the times, as well as along the tube.

In the case of a 2D fluid, the notion of point vortex with zero area is introduced. The velocity field generating such a vortex may be represented by the expressions

$\mathbf{v}=\frac{\kappa}{2 \pi r} \mathbf{e}_{\theta}=\frac{\kappa}{2 \pi}\left(-\mathbf{i} \frac{y}{r^{2}}+\mathbf{j} \frac{x}{r^{2}}\right) ;$

$\mathbf{e}_{\theta}=\mathbf{j} \cos \theta-\mathbf{i} \sin \theta ; \quad \mathbf{e}_{r}=\mathbf{i} \cos \theta+\mathbf{j} \sin \theta$

$\boldsymbol{\nabla}=\mathbf{i} \frac{\partial}{\partial x}+\mathbf{j} \frac{\partial}{\partial y}=\frac{\partial}{\partial r} \mathbf{e}_{r}+\frac{1}{r} \frac{\partial}{\partial \theta} \mathbf{e}_{\theta}$

Here, $\kappa$ is the vortex strength, whereas the unit vectors $\mathbf{i}, \mathbf{j}, \mathbf{e}_{r}$, and $\mathbf{e}_{\theta}$ correspond to the rectangular and polar $2 \mathrm{D}$ coordinates. Following [26], we must take the definition of a curl in the $2 \mathrm{D}$ space into account, namely that the curl of a vector is a scalar and the curl of a scalar is a vector as follows:

$\omega=\operatorname{Curl} \mathbf{v}=\boldsymbol{\nabla} \times \mathbf{v}=\varepsilon^{i j} \partial_{i} v_{j} ; \quad(\operatorname{Curl} S)^{i}=\varepsilon^{i j} \partial_{j} S$, 
where $\varepsilon^{i j}$ is an antisymmetric tensor with the properties $\varepsilon^{12}=-\varepsilon^{21}=1 ; \varepsilon^{11}=\varepsilon^{22}=0$.

These rules lead to the vorticity of a point vortex with the velocity field (15)

$\omega(r)=\operatorname{Curl} \mathbf{v}=\frac{\kappa}{2 \pi} \Delta \ln r=\kappa \delta^{(2)}(\mathbf{r})$.

The velocity field created by a point vortex has a singularity. It is irrotational or potential almost in the whole space except at the origin at the point $r=0$. For this reason, the vortex area is zero. Nevertheless, the summary vorticity due to singularity (17) is finite. In the same way, the circulation of the vortex is also finite as follows:

$\int \omega d^{2} \mathbf{r}=\lim _{r \rightarrow \infty} \oint \frac{\kappa}{2 \pi r} \mathbf{e}_{\theta} d \mathbf{l}=\kappa ; \quad d \mathbf{l}=r d \theta \mathbf{e}_{\theta}$.

A fluid containing a point vortex will have potential flow almost everywhere. A point vortex in an incompressible liquid has the energy

$\int \frac{m v^{2}}{2} d^{2} \mathbf{r}=\frac{m \kappa^{2}}{2 \pi} \ln \frac{R}{a}$

where $R$ is the length scale of the whole system and $a$ is the core radius. A classical system of $N$ point vortices in an incompressible liquid has the kinetic energy associated with each vortex and the interaction energy between them. This interaction does not come from the electric charge of vortices, because they are neutral. For two vortices with guiding centers $\mathbf{R}_{1}$ and $\mathbf{R}_{2}$, it is useful to define a guiding center of a pair $\mathbf{R}_{\mathrm{gc}}$ and its relative coordinate $\mathbf{R}_{\text {rel }}$ in the form

$\mathbf{R}_{\mathrm{gc}}=\mathbf{R}_{1}+\mathbf{R}_{2} ; \quad \mathbf{R}_{\mathrm{rel}}=\mathbf{R}_{1}-\mathbf{R}_{2}$.

The equations of motion for a pair of vortices with equal strengths $\kappa_{1}=\kappa_{2}=\kappa$ are

$\dot{X}_{\mathrm{gc}}=\dot{Y}_{\mathrm{gc}}=0$

$\dot{X}_{\mathrm{rel}}=-\frac{\kappa Y_{\mathrm{rel}}}{\pi R_{\mathrm{rel}}^{2}}$

$\dot{Y}_{\mathrm{rel}}=\frac{\kappa}{\pi} \frac{X_{\mathrm{rel}}}{R_{\mathrm{rel}}^{2}}$

These equations describe a circular motion around a fixed point named as a stationary guiding center with an angular velocity $\Omega$ depending on the constant separation distance of the vortices $\left|\mathbf{R}_{\text {rel }}\right|$ as follows:

$\Omega=\frac{\kappa}{\pi\left|\mathbf{R}_{\mathrm{rel}}\right|^{2}}$.
For a pair of vortices with opposite vorticities $\kappa=$ $\kappa_{1}=-\kappa_{2}$, i.e. for a vortex-antivortex pair, the equations of motion are

$\dot{X}_{\mathrm{gc}}=\frac{\kappa}{\pi} \frac{Y_{\mathrm{rel}}}{\left|\mathbf{R}_{\mathrm{rel}}\right|^{2}} ;$

$\dot{Y}_{\mathrm{gc}}=-\frac{\kappa}{\pi} \frac{X_{\mathrm{rel}}}{\left|\mathbf{R}_{\mathrm{rel}}\right|^{2}} ;$

$\dot{X}_{\text {rel }}=\dot{Y}_{\text {rel }}=0$.

The vortices will not move relative each other, but will follow a straight line perpendicular to the vector $\mathbf{R}_{\text {rel }}$ connecting the vortices [10].

This picture is exactly the same as the structure of a $2 \mathrm{D}$ magnetoexciton moving with wave vector $\mathbf{k}$ perpendicular to the vector $\mathbf{d}$ connecting the electron and the hole in the pair with a constant distance $d=k l^{2}$ at a given k.

Now we will discuss the quantum vortices following $[10,25]$. The existence of quantum vortices was suggested for the first time by Onsager [27], who proposed that the circulation in the superfluid He-II is quantized with the quantum of circulation $h / m$. The quantum vortices in He-II were discussed by Feynman [28], whereas a quantized line was observed by Vinen [29]. The quantization of the vorticity in He-II can be explained in the frame of the GL theory. The velocity field of a superfluid described by the wave function

$\phi=\sqrt{\rho} e^{i S}$

can be written as

$\mathbf{v}=\frac{\hbar}{m} \nabla S$.

The circulation around a close path $C$ becomes

$\kappa=\oint \mathbf{v} d \mathbf{l}=\frac{\hbar}{m} \oint \nabla S d \mathbf{l}=\frac{\hbar}{m} \delta S$,

$\delta S$ is a change in the phase of the wave function, as one moves around the close path $C$. But the wave function must be single-valued. For this reason, $\delta S$ must be an integer multiple of $2 \pi$. This means that

$\kappa=\frac{\hbar}{m} 2 \pi s, \quad s=0, \pm 1, \pm 2 \ldots$

The vorticity of a quantum vortex has discrete values with the quantum $h / m$. This definition of vorticity differs from the classical hydrodynamics, $\boldsymbol{\omega}=\boldsymbol{\nabla} \times \mathbf{v}$. The 
only rotational invariant wave function having property (27), being written in polar coordinates, has $S=s \theta$

$\phi(\mathbf{r})=f(r) e^{i s \theta}$.

It produces the same velocity field as a classical point vortex

$\mathbf{v}=\frac{\hbar s}{m r} \mathbf{e}_{\theta}=\frac{\kappa}{2 \pi r} \mathbf{e}_{\theta}$.

The kinetic energy

$E=\int \frac{1}{2} m v^{2} d^{2} r=\frac{\hbar^{2} \pi}{m} s^{2} \ln (R / \xi)$

is expressed now through the coherence length $\xi$ instead of the core radius $a$. The cutoff at $\xi$ is used to avoid the logarithmic divergence near the vortex core.

Inserting the vortex function (28) into the GinzburgPitaevskii-Gross equation, Myklebust [10, 25] obtained the equation for the function $f(r)$,

$\frac{d^{2} f}{d r^{2}}+\frac{1}{r} \frac{\partial f}{\partial r}+\left(2-\frac{s^{2}}{r^{2}}\right) f-2 f^{3}=0$

depending only on $s^{2}$. As distinct from He-II, the BoseEinstein condensate in superconductors is formed by Cooper pairs with the charge $q=-2 e$ instead of the neutral atoms. The type-II superconductors allow the magnetic field to penetrate in metals forming quantized vortices, while the magnetic field cannot penetrate at all in the type-I superconductors [30]. The quantized vortices do exist in the form of filaments named Abrikosov's lines. They have a mixed electron and electromagnetic field origin and were described for the first time by Abrikosov [31] on the base of the GL theory with a nonzero electromagnetic field A. It was shown that the magnetic flux through a vortex tube is quantized with the flux quantum $\phi_{0}$

$\int \mathbf{B} d \boldsymbol{\sigma}=\oint \mathbf{A} d \mathbf{l}=n \phi_{0} ; \quad \phi_{0}=\frac{2 \pi \hbar c}{|q|}$.

The total energy per unit length of the vortex tube is finite and equals

$E=\left(\frac{\phi_{0}}{4 \pi \lambda}\right)^{2} \ln \frac{\lambda}{\xi} ; \quad \lambda>\xi$,

where $\lambda$ is the penetration length of the magnetic field into the II-type superconductors, as was introduced by F. London and H. London [32], whereas $\xi$ is the correlation length between the electrons in a Cooper pair.
Girvin [18] suggested that the contribution of the electromagnetic field to the resultant current density $\mathbf{j}(\mathbf{r})$ determined in the case of FQHE as

$\mathbf{j}(\mathbf{r})=\frac{1}{2}\left\{\psi^{*}(r)(-i \hbar \nabla) \psi(r)+\psi(r)(i \hbar \nabla) \psi^{*}(r)\right\}+$

$+\frac{e}{c} \mathbf{A} \psi^{*}(r) \psi(r)$

will reorganize the point vortex state in such a way that its resultant circulation at great distances $r \rightarrow \infty$ will be zero

$\oint \mathbf{j}(r) d \mathbf{l}=0$.

This is possible only under the condition that the magnetic flux through the vortex surface will be quantized in the form

$\int \operatorname{rot} \mathbf{A} d^{2} \mathbf{r}=\oint \mathbf{A} d \mathbf{l}=-m \phi_{0} ; \phi_{0}=\frac{2 \pi \hbar c}{|e|}$.

This value, being multiplied by $n_{0}|e| / c$, compensates exactly the circulation arising from the electron part of the current density

$\oint \frac{1}{2}\left\{\psi^{*}(r)(-i \hbar \nabla) \psi(r)+\psi(r)(i \hbar \nabla) \psi^{*}(r)\right\} d \mathbf{l}=2 \pi \hbar m n_{0}$,

since the wave function $\psi(r)$ has the form

$\psi(r)=\sqrt{n_{0}} f(r) e^{i m \theta} ; \quad f(r) \rightarrow 1 ; \quad r \rightarrow \infty$.

The number of magnetic flux quanta $m$ must be opposite to the magnetic quantum number of the electron wave function. The creation energy of such point vortex is finite and no extensive as in the case of a pure electron vortex. As was mentioned by Girvin and MacDonald [19], the isolated vortices cost only a finite energy. They can be excited thermally by one. Earlier, it was necessary to create a vortex-antivortex pair with finite creation energy for a pair as a whole, but with an extensive energy for each of them. Only in the last case, the Kosterlitz-Thouless phase transition is possibly related to the unbinding of the vortices in pairs.

\section{Gauge Transformations and Statistical Gauge Field}

Girvin and MacDonald [19] revealed a hidden symmetry of the Laughlin's ground state wave function [33]describing the FQHE of a $2 \mathrm{D}$ one-component electron gas 
(OCEG). This wave function is

$\psi\left(z_{1}, \ldots, z_{N}\right)=\prod_{i<j}\left(z_{i}-z_{j}\right)^{m} \exp \left[-\frac{1}{4} \sum_{k}\left|z_{k}\right|^{2}\right]$.

The filling factor of the lowest Landau level (LLL) is a fractional number $\nu=1 / m$, with integer $m>1$. $z_{k}=x_{k}+i y_{k}$ are the complex coordinates of particles in the symmetric gauge. Under the interchange of any two particles, the wave function (39) is antisymmetric at odd values of $m$ and symmetric at even values, describing the fermions and the bosons, respectively. But changing the phase of the wave function (39) with the use of a singular gauge transformation

$\psi_{\text {new }}\left(z_{1}, \ldots, z_{N}\right)=$

$=\exp \left[-i m \sum_{i<j} \arg \left(z_{i}-z_{j}\right)\right] \psi\left(z_{1}, \ldots, z_{N}\right)=$

$=\prod_{i<j}\left|z_{i}-z_{j}\right|^{m} \exp \left[-\frac{1}{4} \sum_{k}\left|z_{k}\right|^{2}\right]$,

we have obtained a bosonic-type wave function at any integer values of $m>1$. The off-diagonal matrix elements of the density matrix $\rho\left(z, z^{\prime}\right)$ calculated with function (39) are short-ranged with a characteristic scale given by the magnetic length, whereas those calculated with the wave function (40) $\tilde{\rho}\left(z, z^{\prime}\right)$ have a slowly decreasing behavior with the power law $\left|z-z^{\prime}\right|^{-m / 2}$. The singular gauge density matrix $\tilde{\rho}\left(z, z^{\prime}\right)$ has an off-diagonal longrange order (ODLRO). The physical origin of this difference is related to the presence of the vortices induced around each particle under the influence of the magnetic flux quanta, as was explained by Stormer [34].

The presence of vortices can be demonstrating by a more simple example proposed by Enger [10] with the wave function $\psi(z)$ of two particles depending only on their relative coordinate $z$. It is supposed that $\psi(z)$ obeys the anyon statistics. After the particle interchanging, it becomes

$\psi\left(e^{i \pi} z\right)=e^{i \theta} \psi(z) ; \quad \begin{array}{ll}\theta=\pi(2 n+1) & \text { for fermions, } \\ \theta=2 \pi n & \text { for bosons }\end{array}$

$n=0, \pm 1, \pm 2 \ldots$.

A gauge transformation $e^{i \eta(z)}$ transforms the wave function $\psi(z)$ into another bosonic-type wave function

$e^{i \eta(z)} \psi(z)=\phi(z)=\phi\left(e^{i \pi} z\right)$.
To satisfy this requirement and the equalities

$e^{i \eta\left(e^{i \pi} z\right)} \psi\left(e^{i \pi} z\right)=e^{i \eta(z)} \psi(z)=e^{i \eta\left(e^{i \pi} z\right)} e^{i \theta} \psi(z)$,

the function $\eta(z)$ must satisfy the equation

$\theta=\eta(z)-\eta\left(e^{i \pi} z\right)$

$\eta(z)=-\frac{\theta}{\pi} \arg z=-\frac{\theta}{\pi} \arctan \frac{y}{x}$.

The transformation of the wave function (42) must be accompanied by the transformation of the electromagnetic field $A_{\mu}[10]$

$\frac{e}{\hbar c} A_{\mu} \rightarrow \frac{e}{\hbar c} A_{\mu}+\partial_{\mu} \eta(z)=\frac{e}{\hbar c}\left(A_{\mu}+a_{\mu}\right) ;$

$\mu=0,1,2$.

In such a way side-by-side with the electromagnetic potential $A_{\mu}$, the supplementary gauge potential $a_{\mu}$ created by the vortices appears:

$\frac{e}{\hbar c} a_{\mu}(\mathbf{r})=\partial_{\mu} \eta(\mathbf{r})$

$a_{\mu}(\mathbf{r})=\frac{\hbar c}{e} \partial_{\mu} \eta(\mathbf{r})=-\frac{\hbar c \theta}{\pi e} \partial_{\mu} \arctan \frac{y}{x}$.

The statistical gauge vector potential has the form

$\mathbf{a}=\frac{\hbar c \theta}{\pi e} \operatorname{Curl} \ln r=\frac{\hbar c \theta}{\pi e} \nabla \times \ln r$,

$a_{i}=\frac{\hbar c \theta}{\pi e} \varepsilon^{i j} \partial_{j} \ln r ; \quad i, j=1,2$.

This vector potential is created by the vortices arising near each particle. It leads to the magnetic field strength [26]

$b(r)=\operatorname{Curl} \mathbf{a}(r)=\boldsymbol{\nabla} \times \mathbf{a}(r)=\varepsilon^{i j} \partial_{i} a_{j}=$

$=-\frac{\hbar c \theta}{e \pi} \Delta \ln r=-\frac{2 \hbar c \theta}{e} \delta^{(2)}(\mathbf{r})$

$\frac{\Delta \ln r}{2 \pi}=\delta^{(2)}(\mathbf{r})$

The magnetic flux created by this magnetic field is

$\int b(r) d^{2} \mathbf{r}=-\frac{2 \pi \hbar c \theta}{e}=-\frac{\theta}{\pi} \phi_{0} ; \quad \phi_{0}=\frac{h c}{e}$. 
It equals $-(2 n+1) \phi_{0}$, when the initial particles described by the function $\psi(z)$ are fermions, and $-2 n \phi_{0}$ for the bosonic wave function $\psi(z)$. This result shows that the initial fermion particles, each of them attaching an odd number of flux quanta, transform themselves into a composite bosons described by the new wave function $\phi(z)$ which obeys the Bose statistics. The effective mass $m$ and the charge $e$ remain the same at least in the given approximation, but the composition and the statistics of final quasiparticles are changed. It is said that the electron attached an odd number of flux tubes, though such tubes do not exist in reality. We can say that, in our case, the initial particles are fermions or electrons, whereas the final quasiparticles are bosons. Formula (48) can be generalized to any number of particles, which create, in a common way, the resultant magnetic field

$b(r)=-\frac{2 \hbar c \theta}{e} \sum_{i=1}^{N} \delta^{2}\left(\mathbf{r}-\mathbf{r}_{i}\right)=-\frac{2 \theta \hbar c}{e} \rho(\mathbf{r})$

where $\rho(\mathbf{r})$ is the density of particles.

As was mentioned above, Zhang, Hanson, and Kivelson [20] have generalized the Ginzburg-Landau theory, by introducing the supplementary Chern - Simons [35] term related with the influence of a statistical gauge field into the Lagrangian. The Lagrangian of the GinzburgLandau-Chern-Simons (GLCS) theory in the form presented by Enger [10] looks as

$$
\begin{gathered}
L=i \hbar \phi^{*}\left(\partial_{t}+\frac{i e}{\hbar c}\left(A_{0}+a_{0}\right)\right) \phi+ \\
+\frac{\hbar^{2}}{2 m} \phi^{*}\left(\nabla+\frac{i e}{\hbar c}(\mathbf{A}+\mathbf{a})\right)^{2} \phi- \\
-\frac{\lambda}{2}\left(\phi^{*} \phi-\rho_{0}\right)^{2}+\frac{\mu}{2} e^{\xi \nu \sigma} a_{\xi} \partial_{\nu} a_{\sigma} .
\end{gathered}
$$

Here the following denotations are used: $\xi, \nu, \sigma=0,1,2$; $\partial_{0}=\frac{1}{c} \partial_{t}, \partial_{i}=\left\{\partial_{1}=\partial_{x}, \partial_{2}=\partial_{y}\right\}$. The tensor $\varepsilon^{\xi \nu \sigma}$ has the components different from zero only for different values of $\xi, \nu, \sigma$. They change the signs at any permutations of two indexes as follows:

$\varepsilon^{012}=1, \varepsilon^{021}=-1, \varepsilon^{102}=-1, \varepsilon^{120}=1$ and so on.

The external electromagnetic $2 \mathrm{D}$ vector potential $\mathbf{A}$ and the scalar potential $A_{0}$ are represented as $A_{\xi}=$ $\left(A_{0}, \mathbf{A}\right) . a_{\xi}$ is the statistical gauge potential with three components, two of them $\mathbf{a}=\left(a_{1}, a_{2}\right)$ generate the statistical "magnetic" field, and the third component $a_{0}$ gives rise to the statistical "electric" field. Two parameters $m$ and $e$ of Lagragian (51) are the effective mass and the charge of the final quasiparticles obeying the Bose statistics. They can differ from the mass and the charge of initial particles. The $\lambda$ and $\rho_{0}$ parameters are typical of the GL theory, while $\mu$ is the Chern-Simons parameter [28]. Variations of (51) with respect to $\phi^{*}$ gives

$$
\begin{aligned}
& {\left[i \hbar \partial_{t}-\frac{e}{c}\left(A_{0}+a_{0}\right)\right] \phi=-\frac{\hbar^{2}}{2 m}\left[\nabla+\frac{i e}{\hbar c}(\mathbf{A}+\mathbf{a})\right]^{2} \phi-} \\
& -\lambda\left(\phi^{*} \phi-\rho_{0}\right) \phi
\end{aligned}
$$

It is a nonlinear Schrödinger equation. The variation of (51) with respect to $a_{0}$ gives

$\mu \varepsilon^{i j} \partial_{i} a_{j}=e \phi^{*} \phi=e \rho$,

which can be transcribed as

$\mu \operatorname{Curl} \mathbf{a}=\mu \boldsymbol{\nabla} \times \mathbf{a}=\mu b=e \rho$.

If one compares it with expression (46), the parameter $\mu$ can be determined as

$\mu=-\frac{2 \theta \hbar c}{e^{2}}$.

For the initial fermion particles with $\theta=\pi(2 n+1)$, the parameter $\mu$ of Lagrangian (51) equals $-\frac{2 \pi \hbar c}{e^{2}}(2 n+1)=$ $-(2 n+1) \frac{\phi_{0}}{e}$. The third variation with respect to $a_{i}$ gives

$\mu\left(\nabla a_{0}-\frac{1}{c} \partial_{t} \mathbf{a}\right)=e \mathbf{j}(r)$

where $\mathbf{j}(r)$ is the current density $2 \mathrm{D}$ vector defined as

$\mathbf{j}=\frac{\hbar}{2 m i}\left\{\phi^{*}\left(\nabla-\frac{i e}{\hbar c}(\mathbf{A}+\mathbf{a})\right) \phi-\right.$

$\left.-\phi\left(\boldsymbol{\nabla}+\frac{i e}{\hbar c}(\mathbf{A}+\mathbf{a})\right) \phi^{*}\right\}$.

Equation (57) states that the statistical "electric" field

$\varepsilon=-\nabla a_{0}+\frac{1}{c} \frac{\partial}{\partial t} \mathbf{a}$

is related to the particle current density $\mathbf{j}(r)$. 
The energy density of the GLCS system in a purely static external magnetic field $\left(A_{0}=0\right)$ equals

$E=\frac{\hbar^{2}}{2 m}\left|\left(\boldsymbol{\nabla}-\frac{i e}{\hbar c}(\mathbf{A}+\mathbf{a})\right) \phi\right|^{2}+\frac{\lambda}{2}\left(\rho-\rho_{0}\right)^{2}$.

The simple solution of these equations can be obtained, by setting

$\phi(\mathbf{r})=\sqrt{\rho_{0}} e^{i S(\mathbf{r})}$.

It must obey the equation

$\nabla S+\frac{e}{\hbar c}(\mathbf{A}+\mathbf{a})=0$.

In the case $S=$ const, we have

$\mathbf{A}+\mathbf{a}=0$.

This means that the corresponding magnetic fields $B=\boldsymbol{\nabla} \times \mathbf{A}$ and $b=\boldsymbol{\nabla} \times \mathbf{a}$ cancel each other. The final quasiparticles named composite bosons [36-38] feel no net magnetic field and behave themselves as bosons interacting with one another via the $\phi^{4}$-type interaction. The notion of composite particles (CPs) consisting from electrons and attached magnetic flux quanta was introduced firstly by Wilczek [36].

As was mentioned above, the collective elementary excitations of the described ground state are the plane waves. Their dispersion law has a gap, which means that the system is an incompressible quantum liquid, which cannot be excited by a very small perturbation [20, 21].

Above, we have discussed the case where the initial wave function $\psi(z)$ with the Fermi statistics was transformed into another wave function $\phi(z)$ obeying the Bose statistics, by using a singular gauge transformation. Read [39-42] investigated the system of 2D charged bosons interacting with a transverse magnetic field and between themselves. The filling factor of the LLL was supposed to be one. This means that there is one flux quantum for each particle. Following Read [39-42], it is equivalently to say that there exists one vortex for each particle. In this case, the vortex has a charge of opposite sign in comparison with the initial particles having the Bose and Fermi statistics. Now, the gauge transformation attaching one vortex to each charged boson will create composite particles with the zero resulting charge and with the Fermi statistics. The neutral composite fermions (CFs) will move in the zero magnetic field. Such system can be described in the frame of the Fermi-liquid-theory. Another version was considered by Halperin, Lee, and Read [43]. The starting Hamiltonian describes the electrons forming a $2 \mathrm{D}$ electron gas
(2DEG) with the filling factor $\nu$ of the LLL equal to one half $(\nu=1 / 2)$. Now, for each electron, there are two flux quanta or two vortices, each of them having the charge $-e / 2$ and the Fermi statistics. Two vortices are equivalent to one 2 -fold vortex with charge $-e$ and the Bose statistics. The gauge transformation of the wave function will transform the initial charged electrons into the composite neutral fermions, each of them consisting from one electron and a 2 -fold vortex. The Hamiltonian of the system will be changed because, instead of the external magnetic field, a supplementary gauge magnetic field will appear. Under well-determined conditions, it cancels exactly the external magnetic field. The initial charged fermions were converted in neutral CFs moving in the zero resulting magnetic field. The fictitious Chern-Simons "magnetic" field created by the vortices being averaged in the mean-field approximation cancels exactly the external magnetic field only in statistical sense and under definite conditions. This happens when the mean density of electrons corresponds to the fractional integer filling factor. In the present example with $\nu=1 / 2$, the gauge transformation does not modify the statistics of composite particles (CPs). As was mentioned above, they are neutral CFs in the zero magnetic field. The singular gauge transformations were first introduced by Wilczek [36]. Halperin, Lee, and Read [43] have introduced the electron creation operator $\psi_{e}^{\dagger}(r)$ and the creation operator of the $\mathrm{CP} \psi^{\dagger}(r)$ containing a gauge transformation of the form

$\psi^{\dagger}(r)=\psi_{e}^{\dagger}(r) \exp \left[-i m \int d^{2} \mathbf{r}^{\prime} \theta\left(\mathbf{r}-\mathbf{r}^{\prime}\right) \hat{\rho}\left(\mathbf{r}^{\prime}\right)\right]$,

$\hat{\rho}(\mathbf{r})=\psi_{e}^{\dagger}(r) \psi_{e}(r)=\psi^{\dagger}(r) \psi(r)$.

Here, $\theta\left(\mathbf{r}-\mathbf{r}^{\prime}\right)$ is the angle between the vector $\mathbf{r}-\mathbf{r}^{\prime}$ and the $x$-axis and has the expression

$\theta\left(\mathbf{r}-\mathbf{r}^{\prime}\right)=\arctan \frac{y-y^{\prime}}{x-x^{\prime}}$

There is a nonlocal dependence between the functions $\psi^{\dagger}(r)$ and $\psi_{e}^{\dagger}(r)$. It has a general application and can be applied not only in the case $m=2$, but for any other integer $m=1,2,3, \ldots$ Last two versions concerning charged bosons with $\nu=1$ and electrons with $\nu=1 / 2$, both of them after the gauge transformations, deal with CFs described in the frame of the Fermi-liquid-theory. In both cases at $T=0$, there exists the sharp Fermi sea and the well-defined Fermi wave vector $k_{F}=1 / l$. The creation operator in the coordinate representation (64) can be 
rewritten in the momentum representation as follows:

$\psi^{\dagger}(\mathbf{k})=\int d^{2} \mathbf{r} e^{i \mathbf{k r}} \psi^{\dagger}(\mathbf{r})$

The single-particle elementary excitations appear in the form of fractionally charged vortices. They are fermions and have finite creation energy as was underlined by Girvin [18] and Girvin and MacDonald [19]. Read [39-42] argued that the ground states of the systems under the condition of FQHE with different fractional integer filling factors $\nu=1 / m$ with $m=1,2,3, \ldots$ contain electrons bound to vortices, since such a binding lowers the system's energy. An $m$-fold vortex carries a charge $-e \nu m$ in the fluid, where $e$ is the electron charge, $e=-|e|$. The electron- $m$-vortex composite, named as $\mathrm{CP}$, has a net charge zero at $\nu=1 / \mathrm{m}$ and behaves like a particle in the zero magnetic field. The vortex is sensitive to the density of electrons, which can vary in space and time even when the external magnetic field and the average filling factor are fixed. The $m$-fold vortices are fermions for $m$ odd and bosons for $m$ even. The composite boson particles can undergo the Bose-Einstein condensation (BEC), because it minimizes their "kinetic" energy. Just the BEC of CBs is the interpretation of the Laughlin's states [33]. The origin of the "kinetic" energy is the potential energy of the interaction between particles. In the case of electrons, it is the Coulomb electronelectron interaction which is not cancelled by the gauge transformation and the CS gauge potential. It is named as "kinetic," because it depends on the wave vector of operators (66). The bound objects such as CPs do, in fact, have such an effective "kinetic" energy. There is an attraction between an electron and an $m$-fold vortex. For the electron, it plays the role of a correlation quasihole. As was shown in (66), the CPs may exist in the form of plane waves, and the many-particle wave functions also can be characterized by the wave vector $\mathbf{k}$. A CP with $\mathbf{k}=0$ would have the electron exactly at the zeroes of the wave function or at the center of the vortex, whereas the CP with the wave vector $|k| \neq 0$ has the electron displaced by the distance $|k| l^{2}$ from their center. One can say that the electron and its correlation quasihole or, in another words, the electron and the $m$-fold vortex experience a potential $V(|k|)$ due to the Coulomb interaction of the electron with other electrons excluded from the vortex core. All these interactions take place in the presence of the neutralizing background. The electron and the $m$-fold vortex experience the magnetic field of the same strength. The both components of the pair will drift in the same direction perpendicular to the vector connecting their centers, so that their separation re- mains constant and equal to $|k| l^{2}$. The energy of a pair is $V(|k|)$, and its group velocity is $\partial V(|k|) / \partial|k|[39-42]$. We can add that this picture coincides with the structure of a 2D magnetoexciton, where the energy $V(|k|)$ equals the expression $E(|k|)[43]$

$$
\begin{aligned}
& E(|k|)=2 \sum_{Q} W_{Q} \sin ^{2}\left(\frac{[\mathbf{Q} \times \mathbf{k}]_{z} l^{2}}{2}\right) ; \\
& W_{Q}=\frac{2 \pi e^{2}}{\varepsilon_{0} S|\mathbf{Q}|} e^{-Q^{2} l^{2} / 2}
\end{aligned}
$$

Here, $\varepsilon_{0}$ is the dielectric constant, and $S$ is the layer surface area.

Girvin, MacDonald, and Platzman [21] elaborated the theory of the collective elementary excitation spectrum in the case of FQHE, closely analogously to Feynman's theory of superfluid helium. The predicted spectrum has a gap at $k=0$ and a deep magneto-roton minimum at a finite wavevector, which is a precursor to the gap collapse associated with the Wigner instability in crystals. They supposed the existence of only one branch of the spectrum of collective elementary excitations. In this approximation named as the single mode approximation (SMA), they have constructed the wave functions of the excited states $\phi_{k}$ acting with the operator of particle density $\hat{\rho}_{k}$ on the ground-state wave function $\psi_{g}$ in the form $\phi_{k}=\hat{\rho}_{k} \psi_{g}$. They determined the energy of the excited state $\Delta(k)$ as

$$
\begin{aligned}
& \Delta(k)=\frac{\left\langle\phi_{k}\left(H-E_{0}\right) \phi_{k}\right\rangle}{\left\langle\phi_{k} \phi_{k}\right\rangle}= \\
& =\frac{\left\langle\psi_{g} \rho_{k}^{\dagger}\left[H_{0}, \rho_{k}\right] \psi_{g}\right\rangle}{\left\langle\psi_{g} \rho_{k}^{\dagger} \rho_{k} \psi_{g}\right\rangle}=\frac{f(k)}{s(k)},
\end{aligned}
$$

where $f(k)$ is the oscillator strength, and $s(k)$ is the static structure factor. The total oscillator strength sum is saturated by the cyclotron mode contribution, and $f(k)$ has a dependence of the type $|k|^{4}$. As was established by Lee and Zhang [45], the contributions of quantum vortices to the dynamical and static structure factors are important. This leads to the dependence $s(k) \sim|k|^{4}$ as $k \rightarrow 0$. In this case, $\Delta(k)$ has a gap. Neglecting the influence of quantum vortices, the dependence $s(k)$ is proportional to $|k|^{2}$, and the energy spectrum is gapless $\Delta(k) \approx k^{2}$ as $k \rightarrow 0$ as the Goldstone mode [46]. In conclusion, for the FQHE in the case $\nu<1$ with a fractionally filled Landau level, the Pauli principle no longer excludes low-lying intra-Landau-level 
excitations. They exist side-by-side with inter-Landaulevel excitations. The last excitations have a cyclotron energy gap [47].

We are studying a coplanar electron-hole (e-h) system with electrons in the conduction band and with holes in the valence band in a strong perpendicular magnetic field. Earlier, such a system was studied in a series of papers $[1-8,44]$. The most of them were devoted to the theory of $2 \mathrm{D}$ magnetoexcitons. But there were the papers devoted to another aspects of these systems. For example, MacDonald, Rezayi, and Keller [48], as well as Joglekar and MacDonald [49], have discussed the photoluminescence (PL) spectrum in the FQHE regime. It was mentioned that the PL spectrum does not exhibit anomalies associated with the FQHE. However, when the electron and hole layers were separated, a new peak in the PL spectrum appears, when the filling factor exceeds a fraction $\nu_{0}$ at which an incompressible quantum liquid occurs. The new peak is separated from the main spectral features by the quasiparticle-quasihole gap. We are interested in the distribution of flux quanta in the case of an $e-h$ system with equal average numbers of electrons and holes $\bar{N}_{e}=\bar{N}_{h}$ with filling factor $\nu=\bar{N}_{e} / N$, where $N$ is the total number of flux quanta $N=\frac{S}{2 \pi l^{2}}, S$ is the layer surface area, and $2 \pi l^{2}$ is the area of the cyclotron orbit. In the case of the fractional filling factor, there is an integer number of flux quanta per each $e-h$ pair. In this case, the creation of vortices has not been studied till now. But one can expect that, in the case of magnetoexcitons, they will be neutral, whereas, in the case of pure electron and pure hole vortices, their "magnetic" gauge fields will compensate each other, so that the charge, statistics of particles, and external magnetic field will remain the same in the meanfield approximation with equal densities of electrons and holes.

Nevertheless, due to quantum fluctuations and to the deviations of the electron and hole densities in space and time from their average values, one can expect the influence of pure electron and hole quantum vortices on the physics of magnetoexcitons side-by-side with the influence of neutral quantum vortices formed by the magnetoexcitons themselves. The last quantum vortices determine the Berezinskii-Kosterlitz-Thouless phase transition $[50,51]$.

1. I.V. Lerner and Yu.E. Lozovik, Zh. Eksp. Teor. Fiz. 78, 1167 (1980).

2. I.V. Lerner and Yu.E. Lozovik, J. Low Temper. Phys. 38, 333 (1980).
3. I.V. Lerner and Yu.E. Lozovik, Sov.Phys.-JETP 53, 763, (1981)].

4. A.B. Dzyubenko and Yu.E. Lozovik, Sov. Phys. Solid State 25, 874 (1983); 26, 938 (1984)]; J. Phys. A 24, 415 (1991).

5. D. Paquet, T.M. Rice, and K. Ueda, Phys. Rev. B 32, 5208 (1985); T.M. Rice, D. Paquet, and K. Ueda, Helv. Phys. Acta 58, 410 (1985).

6. S.A. Moskalenko and D.W. Snoke, Bose-Einstein Condensation of Excitons and Biexcitons and Coherent Nonlinear Optics with Excitons (Cambridge Univ. Press, Cambridge, 2000).

7. S.A. Moskalenko, M.A. Liberman, P.I. Khadzhi, E.V. Dumanov, Ig.V. Podlesny, and V. Botan, Sol. State Comm. 140/5, 236 (2006); S.A. Moskalenko, M.A. Liberman, P.I. Khadzhi, E.V. Dumanov, Ig.V. Podlesny, and V. Botan, Physica E 39/1, 137 (2007).

8. S.A. Moskalenko, M.A. Liberman, E.V. Dumanov, J. of Nanoelectron. and Optoelectron. 4, 52 (2009).

9. E. Prange and S.M. Girvin, The Quantum Hall Effect, (Springer, New York, 1986).

10. H. Enger, Vortices in Chern-Simons-Ginzburg-Landau Theory and the Fractional Quantum Hall Effect, Thesis submitted to the degree of Candidatus Scientiarum (Univ. of Oslo, Oslo, 1998).

11. L.D. Landau and E.M. Lifshitz, Statistical Physics (Pergamon, Oxford, 1969).

12. V.L. Ginzburg and L.D. Landau, Zh. Eksp. Teor. Fiz. 20, 1064 (1950).

13. V.L. Ginzburg and L.P. Pitaevskii, Zh. Eksp. Teor. Fiz. 34, 1240 (1958).

14. L.P. Pitaevskii, Sov. Phys. JETP 12, 155 (1961).

15. E.P. Gross, Nuovo Cimento 20, 454 (1961).

16. P. Nozieres and D. Pines, The theory of Quantum Liquids (Addison-Wesley, New York, 1990).

17. N.N. Bogoliubov, Izv. Akad. Nauk SSSR Ser. Fiz. 11, 77 (1947), Collection of papers in three volumes (Naukova Dumka, Kiev, 1971), Vol. 2 and 3 (in Russian).

18. S.M. Girvin, The quantum Hall effect: Novel Excitations and Broken-Symmetries (Indiana Univ., Bloomington, 1998).

19. S.M. Girvin and A.H. MacDonald, Phys. Rev. Lett. 58, 1252 (1987).

20. S.C. Zhang, T.H. Hanson, and S. Kivelson, Phys. Rev. Lett. 62, 82 (1989).

21. S.M. Girvin, A.H. MacDonald, and P.M. Platzman, Phys. Rev. Lett. 54, 581 (1985); Phys. Rev. B 33, 2481 (1986).

22. R.B. Laughlin, Phys. Rev. Lett. 60, 2677 (1988).

23. S. Kivelson, C. Kallin, D.P. Arovas, and J. Schrieffer, Phys. Rev. Lett. 56, 873 (1986). 
24. D.H. Lee, G. Baskaran, and S. Kivelson, Phys. Rev. Lett. 59, 2467 (1987).

25. L.K. Myklebust, Quantized Vortices, Cand. Scient. Thesis, University of Oslo, 1996.

26. R. Jackiw and So Young Pi, Phys. Rev. D 42, 3500 (1990).

27. L. Onsager, Nuovo Cimento 6, Suppl. 2, 249 (1949).

28. R.P. Feynman, in Progress in Low Temperature Physics, edited by C.J. Gorter (North-Holland, Amsterdam, 1955), Vol. 1, p.17.

29. W.F. Vinen, Nature 181, 1524 (1958); Proc. R. Soc. A 260, 218 (1961).

30. P.G. De Gennes, Superconductivity of Metals and Alloys (Benjamin, New-York, 1966).

31. A.A. Abrikosov, Sov. Phys. JETP 5, 1174 (1957).

32. F. London, Superfluids (Wiley, New York, 1950).

33. R.B. Laughlin, Phys. Rev. Lett. 50, 1395 (1983).

34. H.L. Stormer, Rev. Mod. Phys. 71, 875 (1999).

35. S.S. Chern and J. Simons, Proc. Nat. Acad. Sci. USA 68, 791 (1971).

36. F. Wilczek, Phys. Rev. Lett. 48, 1144 (1982); 49, 957 (1982).

37. R.B. Laughlin, Rev. Mod. Phys. 71, 863 (1999).

38. J.K. Jain. Composite Fermions (Cambridge Univ. Press, Cambridge, 2007).

39. N. Read, Phys. Rev. Lett. 62, 86 (1989).

40. N. Read, Semicond. Sci. Techn. 9, 1859 (1994).

41. N. Read, arxiv. Cond-mat (9501090V), 19 Jan., 1995.

42. N. Read, Phys. Rev. B 58, 16262 (1998).

43. B.L. Halperin, P.A. Lee, and N. Read, Phys. Rev. B 47, 7312 (1993).
44. S.A. Moskalenko, M.A. Liberman, D.W. Snoke, and V.V. Botan, Phys. Rev. B 66, 245316 (2002).

45. D.H. Lee and S.C. Zhang, Phys. Rev. Lett. 66, 1220 (1991).

46. J. Goldstone, Nuovo Cimento 19, 154 (1961).

47. C. Kallin and B.I. Halperin, Phys. Rev. B 30, 5655 (1984).

48. A.H. MacDonald, E.A. Rezayi, and D. Keller, Phys. Rev. Lett. 68, 1939 (1992).

49. Y.N. Joglekar and A.H. MacDonald, Phys. Rev. B 64, 155315 (2001).

50. V.L. Berezinskii, JETP 59, 907 (1970).

51. J.M. Kosterlitz and D.J. Touless, J. Phys. C 6, 1181 (1973).

Received 05.07.11

ДВОВИМІРНІ МАГНІТОЕКСИТОНИ В УМОВАХ

ДРОБОВОГО КВАНТОВОГО ЕФЕКТУ ХОЛЛА

С.А. Москаленко, М.А. Ліберман, Б.В. Новиков,

Е.С. Киселъова, Є.В. Думанов, Ф. Чербу

Р е $з$ ю м е

Компланарні електрони і дірки в сильному перпендикулярному магнітному полі за низьких температур утворюють магнітоекситони, якщо кулонівська взаємодія між електронами і дірками на нижчих рівнях Ландау відіграє основну роль. Але якщо шари з електронами та дірками розділені просторово і кулонівська електрон-діркова взаємодія слабшає, утворюються двовимірні електронний і дірковий гази. Їх властивості в умовах дробового квантового ефекту Холла можуть впливати на властивості двовимірних магнітоекситонів. Ці властивості обговорено в даній роботі. 